\title{
Intercalated Graphene Bilayers: London Penetration Depth Study
}

\author{
K.M. Skoczylas ${ }^{a, *}$, A.E. AuguściK ${ }^{b}$ AND R. SzCZĘŚNIAK ${ }^{b}$ \\ a Jan Długosz University in Częstochowa, Institute of Physics, \\ al. Armii Krajowej 13/15, 42-200 Częstochowa, Poland \\ ${ }^{b}$ Częstochowa University of Technology, Institute of Physics, \\ al. Armii Krajowej 19, 42-200 Częstochowa, Poland \\ Doi: 10.12693/APhysPolA.138.178 \\ *e-mail: kamil.skoczylas@ajd.czest.pl
}

\begin{abstract}
The Eliashberg strong-coupling theory of superconductivity has been used to obtain the London penetration depth functions $\left(\lambda_{\mathrm{L}}\right)$ for superconducting doped graphene bilayers. Analyzed systems can be described by the following chemical formula: $\mathrm{C}_{6} \mathrm{XC}_{6}$, where $\mathrm{X}$ is the dopant belonging to the I or II group (either $\mathrm{K}, \mathrm{Rb}$, or $\mathrm{Ca}, \mathrm{Sr}$ ). Our recent work on these systems show the capability of superconducting state occurrence with the highest critical temperature $\left(T_{c}\right)$ for calcium intercalation $\left(T_{c}=14.56 \mathrm{~K}\right)$. This result adds to the very long list of eligible characteristics of doped two-dimensional graphene sheet. This work comprises the capability of $\mathrm{C}_{6} \mathrm{XC}_{6}$ systems to attenuate the magnetic vortices arising inside the structure of the superconductor under the application of an external magnetic field or electric current. We note the highest $\lambda_{\mathrm{L}}$ (at $T=0 \mathrm{~K}$ ) values for the case of calcium intercalation which holds the highest $T_{c}$. However, we also observe that rubidium insertion does not lead to higher $\lambda_{\mathrm{L}}(0)$ value than potassium and strontium intercalation $\left(T_{c}=8.67 \mathrm{~K}\right.$ and $8.74 \mathrm{~K}$, respectively) with the lowest critical temperature $\left(T_{c}=5.47 \mathrm{~K}\right)$. Additionally, the course of $\left(\lambda_{\mathrm{L}}(0) / \lambda_{\mathrm{L}}(T)\right)^{2}$ function can be compared to the BCS theory predictions which lead to the conclusion of $\mathrm{C}_{6} \mathrm{XC}_{6}$ group being conventional electronphonon superconductors with properties beyond the BCS theory.
\end{abstract}

topics: superconductivity, two-dimensional, graphene, Eliashberg theory, thermodynamic properties

\section{Introduction}

Since first time synthesized, graphene occupies the center of interest of two-dimensional materials due to superior physical and electronic properties of carbon atomic layer which is made of. These, allowing graphene to be seen as the material of the future, do not include the superconducting state occurrence within pristine carbon monolayer. Notwithstanding, two-dimensional scheme, apart of letting the material to possess peculiar characteristics, also allows for almost unrestricted structure doping and arrangement. In such manner, lithium atoms deposition in the 1:6 ratio $\left(\mathrm{LiC}_{6}\right)$ on the carbon sheet make the system drop electric resistance under the critical temperature $\left(T_{c}\right)$ of $5.9 \mathrm{~K}$ [1]. Similar result can be observed in Ca intercalated graphene bilayer and pristine, twisted graphene bilayer $[2,3]$. Moreover, density functional theory calculations also lead to the conclusion of superconducting state occurrence in $\mathrm{C}_{6} \mathrm{CaC}_{6}$ system with higher critical temperature, between $6.8 \mathrm{~K}$ and $8.1 \mathrm{~K}$ [4].

Having in mind the above observations, recently, we have done the study of electron-phonon structure of intercalated graphene bilayer with the I and II group atoms (K, $\mathrm{Ca}, \mathrm{Rb}$, and $\mathrm{Sr}$, respectively) within the $1: 12$ ratio $\left(\mathrm{C}_{6} X \mathrm{C}_{6}\right)$ [5]. Complimentary, we have calculated the superconducting energy gap functions and critical temperature values for the aforementioned systems.
In this paper we focus on the London penetration depth functions $\left(\lambda_{\mathrm{L}}\right)$ which describes the length for which magnetic vortices appearing in the superconductor exponentially attenuate. In fact, high enough magnetic field or electric current applied to the material lead to break of the superconducting state because of expanding magnetic vortices inside material.

Our method to this issue employs the strongcoupling Eliashberg theory of superconductivity, allowing to attain the thermodynamic properties of the superconducting state. The main reason for this approach are high electron-phonon coupling constant values $\left(\lambda^{\mathrm{EPC}}\right)$ for $\mathrm{C}_{6} \mathrm{KC}_{6}, \mathrm{C}_{6} \mathrm{CaC}_{6}, \mathrm{C}_{6} \mathrm{RbC}_{6}$, and $\mathrm{C}_{6} \mathrm{SrC}_{6}$ systems $(0.86,1.18,0.93,0.85$, respectively), obtained in our previous work together with the Eliashberg spectral functions $\alpha^{2} F(\Omega)$, being the main input for further computations.

\section{Computational method}

The Eliashberg theory of superconductivity utilizes the equations for the order parameter $\phi(\omega)$ and the wave function renormalization factor $Z(\omega)$ functions [6]. The imaginary part $\phi_{n} \equiv \phi\left(i \omega_{n}\right)$ and $Z_{n} \equiv Z\left(\mathrm{i} \omega_{n}\right)$ takes the following shape [7]:

$\phi_{m}=\frac{\pi}{\beta} \sum_{n=-M}^{M} \frac{\lambda\left(\mathrm{i} \omega_{m}-\mathrm{i} \omega_{n}\right)-\mu^{*} \theta\left(\omega_{c}-\left|\omega_{n}\right|\right)}{\sqrt{\omega_{n}^{2} Z_{n}^{2}+\phi_{n}^{2}}} \phi_{n}$ 
for order parameter function, whereas wave function renormalization factor is defined as:

$$
Z_{m}=1+\frac{1}{\omega_{m}} \frac{\pi}{\beta} \sum_{n=-M}^{M} \frac{\lambda\left(\mathrm{i} \omega_{m}-\mathrm{i} \omega_{n}\right)}{\sqrt{\omega_{n}^{2} Z_{n}^{2}+\phi_{n}^{2}}} \omega_{n} Z_{n} .
$$

The Matsubara frequency $\left(\omega_{m}\right)$ is defined as: $\omega_{m} \equiv$ $(\pi / \beta)(2 m-1)$, where $m=0, \pm 1, \pm 2, \ldots, \pm M$ for the maximum value of $M=1100$, assuring high computational precision, also $\beta \equiv\left(k_{\mathrm{B}} T\right)^{-1}$. Depairing effects of the Cooper pair are taken into consideration as the $\mu^{*} \theta\left(\omega_{c}-\left|\omega_{n}\right|\right)$ term, involving the Coulomb interactions where $\mu^{*}$ (0.15 in this work) is the Coulomb pseudopotential and $\theta$ is the Heaviside step function [8]. On the other hand, pairing kernel is referred to as

$$
\lambda(z) \equiv 2 \int_{0}^{\Omega_{\max }} \mathrm{d} \Omega \frac{\Omega}{\Omega^{2}-z^{2}} \alpha^{2} F(\Omega) .
$$

As it was mentioned, we take the Eliashberg functions $\alpha^{2} F(\Omega)$ for studied systems from our recent work [5]. Basing on those, we take the maximum phonon frequency value $\Omega_{\max }=24 \mathrm{meV}$, also the cessation frequency $\omega_{c}=10 \Omega_{\max }$.

The London penetration depth function within the Eliashberg approach can be derived from the following relation:

$\frac{1}{e^{2} \nu_{\mathrm{F}}^{2} N\left(\varepsilon_{\mathrm{F}}\right) \lambda_{\mathrm{L}}^{2}(T)}=\frac{4 \pi}{3 \beta} \sum_{m=1}^{M} \frac{\Delta_{m}^{2}}{Z_{m}^{S}\left[\omega_{m}^{2}+\Delta_{m}^{2}\right]^{3 / 2}}$,

where $e$ is the electron electric charge, $\nu_{F}$ and $N\left(\varepsilon_{\mathrm{F}}\right)$ terms are the Fermi velocity and the electron population function on the Fermi level, respectively, also $\Delta_{m}=\phi_{m} / Z_{m}$. Furthermore, $\lambda_{\mathrm{L}}$ for $T=0 \mathrm{~K}$ is defined as: $\lambda_{\mathrm{L}}(0)=e \nu_{\mathrm{F}} \sqrt{N\left(\varepsilon_{\mathrm{F}}\right)} \lambda_{\mathrm{L}}(T)$ and gives the insight on the maximum London penetration depth value for given superconductor.

\section{Numerical results}

Both order parameter and wave function renormalization factor together with the superconducting energy gap functions were calculated in our preliminary work. These were crucial to attain the critical temperature values for $\mathrm{C}_{6} \mathrm{KC}_{6}, \mathrm{C}_{6} \mathrm{CaC}_{6}, \mathrm{C}_{6} \mathrm{RbC}_{6}$, and $\mathrm{C}_{6} \mathrm{SrC}_{6}$ systems which can be seen in Table $\mathrm{I}$.

At this point, we were able to compute the function dependent on the London penetration depth (see Eq. (4)). For the plot clarity and readability we draw squared reduced London penetration depth function $\left(\lambda_{\mathrm{L}}(0) / \lambda_{\mathrm{L}}(T)\right)^{2}$ on the reduced temperature $\left(T / T_{c}\right)$, which is common way to visualize and compare this curve (see Fig. 1) [9].

It can be clearly seen that $\left(\lambda_{\mathrm{L}}(0) / \lambda_{\mathrm{L}}(T)\right)^{2}$ functions diverge from the BCS theory predictions in all cases. Interestingly, highest critical temperature obtained for highest $\lambda^{\mathrm{EPC}}$ (see Introduction section) value (Ca doping) lies closest to the weakcoupling theory predictions. Complementing this observation with the data given in Table I, we can say that London penetration depth function depends mostly on the wave function renormalization
TABLE I

London penetration depth at $T=0 \mathrm{~K}$, critical temperature values and $T_{c} / \omega_{l n}$ ratio obtained for studied $\mathrm{C}_{6} \mathrm{XC}_{6}$ systems, complemented with the superconducting energy gap and wave function renormalization factor numbers for first Matsubara frequency, at zero temperature from our previous work [5]

\begin{tabular}{c|c|c|c|c|c}
\hline \hline$X$ & $\begin{array}{c}\lambda_{\mathrm{L}}(0) \\
{[\text { arb.u.] }}\end{array}$ & $T_{c} / \omega_{l n}$ & $\begin{array}{c}T_{c} \\
{[\mathrm{~K}]}\end{array}$ & $\begin{array}{c}Z(0)_{m=1} \\
{[\mathrm{meV}]}\end{array}$ & $\begin{array}{c}\Delta(0)_{m=1} \\
{[\mathrm{meV}]}\end{array}$ \\
\hline $\mathrm{K}$ & 1.66 & 0.038 & 8.67 & 1.82 & 1.43 \\
$\mathrm{Ca}$ & 1.79 & 0.087 & 14.56 & 2.09 & 2.53 \\
$\mathrm{Rb}$ & 1.69 & 0.046 & 5.47 & 1.88 & 0.91 \\
$\mathrm{Sr}$ & 1.66 & 0.037 & 8.74 & 1.81 & 1.43
\end{tabular}

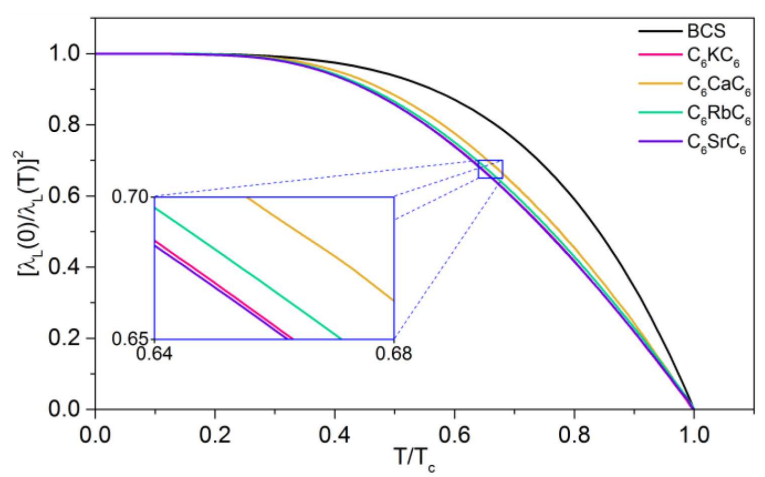

Fig. 1. Obtained squared reduced London penetration depth functions for studied systems compared to function trace determined by BCS theory [10].

factor, similarly to the $\lambda_{\mathrm{L}}(0)$ value. The $T_{c} / \omega_{l n}$ ratio (where $\omega_{l n}$ is the characteristic phonon frequency of a system) is closely related to the superconducting coupling strength and for studied structures, obtained values reproduce the course of $\left(\lambda_{\mathrm{L}}(0) / \lambda_{\mathrm{L}}(T)\right)^{2}$ functions with satisfying precision comparing to the results of Carbotte [9]. To make this clear, for every $T_{c} / \omega_{l n}$ value we get different course of the $\left(\lambda_{\mathrm{L}}(0) / \lambda_{\mathrm{L}}(T)\right)^{2}$ function: for $T_{c} / \omega_{l n}=0$ BCS theory predicted plot can be made and for $T_{c} / \omega_{l n}>0$ reduced London penetration depth functions diverge vastly.

For the better view on the discrepancy between theoretical BCS value we have plotted the $\left(\lambda_{\mathrm{L}}(0) / \lambda_{\mathrm{L}}(T)\right)^{2}-\chi$ function, where $\chi=1-t^{4}$ is the BCS theory function (see Fig. 2).

It is counterintuitive to see that for better thermodynamic properties of superconducting state in the material that has superior (relatively) critical temperature we observe greater London penetration depth. This means that magnetic vortices occurring within the material will occupy greater area under the same applied current or external magnetic field as for other studied materials, thus breaking the superconducting state more easily. 


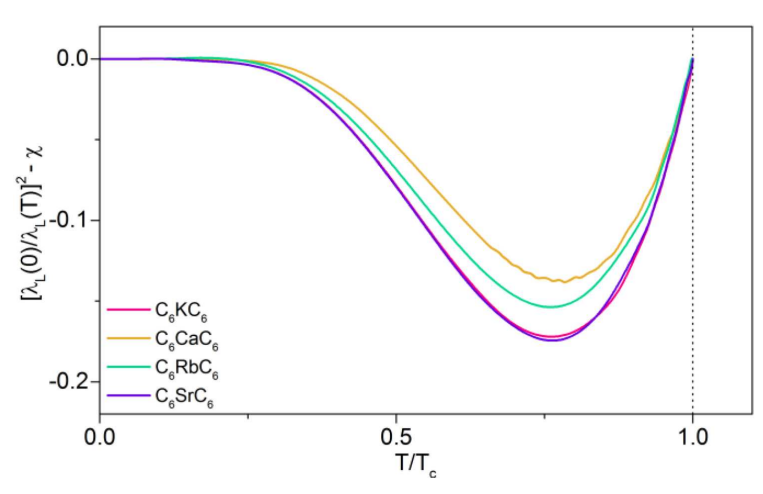

Fig. 2. The difference between squared reduced London penetration depth functions and BCS theoretical values.

\section{Conclusions}

In this paper we discuss the influence of different atoms intercalation to the graphene bilayer on the London penetration depth functions. These were obtained using the strong-coupling Eliashberg theory of superconductivity. We show that BCS theory cannot accurately determine the London penetration depth function course in case of studied $\mathrm{C}_{6} X \mathrm{C}_{6}$ group. Additionally, results indicate deviation in $\left(\lambda_{\mathrm{L}}(0) / \lambda_{\mathrm{L}}(T)\right)^{2}$ functions proportional to the $Z(0)_{m=1}$ values.

The London penetration depth value at $T=0 \mathrm{~K}$ show the peculiar tendency of increment together with the critical temperature. At first glance this effect is counterintuitive, due to the fact of better thermodynamic properties of the superconducting state for the materials having higher $T_{c}$. However, similar results were obtained by Kim in his Ph.D. thesis [11], where iron content has been changed with platinum $\left(\mathrm{Ca}_{10}\left(\mathrm{Pt}_{3} \mathrm{As}_{8}\right)\left(\left(\mathrm{Fe}_{1-x} \mathrm{Pt}_{x}\right)_{2} \mathrm{As}_{2}\right)_{5}\right.$ compound). During such procedure London penetration depth value at zeroth temperature changed to maximum for middle Pt doping and critical temperature, whereas diminished for highest platinum content and $T_{c}$. Kim concludes this effect to be potentially explained by an increasing anisotropy of the superconducting gap during doping. Unfortunately, we are unable to determine on what basis this effects occurs, thus we leave this issue open for the discussion.

\section{References}

[1] B.M. Ludbrook, G. Levy, P. Nigge et al., Proc. Natl. Acad. Sci. 112, 38 (2015).

[2] J. Chapman, Y. Su, C.A. Howard, D. Kundys, A.N. Grigorenko, F. Guinea, A.K. Geim, I.V. Grigorieva, R.R. Nair, Sci. Rep. 6, 23254 (2016).

[3] Y. Cao, V. Fatemi, A. Demir et al., Nature 556, 7699 (2018).

[4] E.R. Margine, H. Lambert, F. Giustino, Sci. Rep. 6, 21414 (2016).

[5] A.P. Durajski, K.M. Skoczylas, R. Szczęśniak, Phys. Chem. Chem. Phys. 21, 5925 (2019).

[6] F. Marsiglio, M. Schossmann, J.P. Carbotte, Phys. Rev. B 37, 4965 (1988).

[7] G. Eliashberg, Sov. Phys. JETP 11, 696 (1960).

[8] P. Morel, P.W. Anderson, Phys. Rev. 125, 1263 (1962).

[9] J.P. Carbotte, Rev. Mod. Phys. 62, 1027 (1990).

[10] J. Bardeen, L.N. Cooper, J.R. Schrieffer, Phys. Rev. 108, 1175 (1957).

[11] H. Kim, Ph.D. Thesis, 2013. 